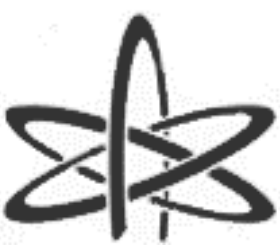

BJRS
BRAZILIAN JOURNAL

$\mathrm{OF}$

RADIATION SCIENCES

09-02A (2021) 01-11

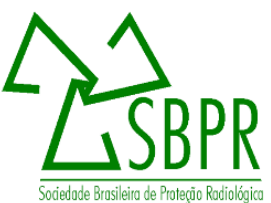

\title{
Solution for the Multigroup Neutron Space Kinetics Equations by Source Iterative Method
}

\author{
Matheus Gularte Tavares, Claudio Zen Petersen, Marcelo Schramm, Rodrigo \\ Zanette
}

Universidade Federal de Pelotas

matheus.gulartetavares@gmail.com

\begin{abstract}
In this work, we used a modified Picard's method to solve the Multigroup Neutron Space Kinetics Equations (MNSKE) in Cartesian geometry. The method consists in assuming an initial guess for the neutron flux and using it to calculate a fictitious source term in the MNSKE. A new source term is calculated applying its solution, and so on, iteratively, until a stop criterion is satisfied. For the solution of the fast and thermal neutron fluxes equations, the Laplace Transform technique is used in time variable resulting in a first order linear differential matrix equation, which are solved by classical methods in the literature. After each iteration, the scalar neutron flux and the delayed neutron precursors are reconstructed by polynomial interpolation. We obtain the fluxes and precursors through Numerical Inverse Laplace Transform by Stehfest method. We present numerical simulations and comparisons with available results in literature.
\end{abstract}

Keywords: Neutron Diffusion Equation, Source Iterative Method, Laplace Transform, Stehfest Algorithm, Polynomial Interpolation.

ISSN: 2319-0612

Accepted: 2021-06-03 


\section{INTRODUCTION}

The iterative source method has been applied with great success in the solution of the neutron transport equation (WILLERT, 2014) (ADAMS, 2012) (DANIELLA, 2017) and in the solution of the steady-state diffusion equation by (ZANETTE, 2017). In the last years many works have been developed in the search for solutions to the problem of kinetic neutron diffusion equation among them, it is possible to emphasize the works (NAHLA, 2012) (CEOLIN, 2011) (CORNO, 2008). In this context, the iterative source method has been used to solve the diffusion kinetic equation. The method consists of estimate an initial distribution for the source term of the fast flux equation decoupling the system and getting it possible to solve the equations separately. It should be noted that this occurs only in systems without up scattering. To solve the MNSKE, the authors apply the Laplace Transform, which transformed the set of partial differential equations into a set of ordinary differential equations (ODE's). These ODE's are solved from classical methods present in the literature. To return the functions to the frequency domain for time domain a numeric inversion of the Laplace was used for. After each iterative process, the term source is updated with the previous expressions of neutron fluxes and delayed neutron precursors. Due to the use of the numerical inverse transform, it is necessary to reconstruct the fluxes and concentrations through an interpolation. The authors prefer to use a polynomial interpolation in order to always maintain a standard structure for all iterations. This iterative process continues until a stop criterion is established.

\section{SPATIAL KINETICS EQUATIONS OF THE MULTIGROUP NEU- TRON DIFFUSION THEORY}

Starting from the Neutron Diffusion Spatial Kinetics equation for two energy groups, six groups of delayed neutron precursors, nuclear parameters not dependent on time and space, without external source and one-dimensional Cartesian geometry we have: 


$$
\begin{aligned}
& \frac{1}{v_{1}} \frac{\partial \phi_{1}(x, t)}{\partial t}-\nabla^{2} D_{1} \phi_{1}(x, t)+\Sigma_{\mathrm{R} 1} \phi_{1}(x, t) \\
& =(1-\beta)\left[v_{1} \Sigma_{\mathrm{f} 1} \phi_{1}(x, t)\right]+\underbrace{(1-\beta)\left[v_{2} \Sigma_{\mathrm{f} 2} \phi_{2}(x, t)\right]+\sum_{i=1}^{6} \lambda_{i} C_{i}(x, t)}_{W}, \\
& \frac{1}{v_{2}} \frac{\partial \phi_{2}(x, t)}{\partial t}=\nabla^{2} D_{2} \phi_{2}(x, t)+\Sigma_{\mathrm{a} 2} \phi_{2}(x, t)+\Sigma_{\mathrm{s} 2} \phi_{1}(x, t) \\
& \frac{\partial C_{i}(x, t)}{\partial t}=\beta_{i}\left[v_{1} \Sigma_{\mathrm{f} 1} \phi_{1}(x, t)+v_{2} \Sigma_{\mathrm{f} 2} \phi_{2}(x, t)\right]-\lambda_{i} C_{i}(x, t)
\end{aligned}
$$

with $i=1, \ldots, 6$, subject to the following boundary conditions:

$$
\begin{aligned}
& \alpha_{1} \phi(0, t)+\beta_{1} \frac{\partial \phi(0, t)}{\partial x}=0, \\
& \alpha_{2} \phi(0, t)+\beta_{2} \frac{\partial \phi(0, t)}{\partial x}=0,
\end{aligned}
$$

where $W$ represents the source term of the fast flux equation. This system of equations is solved through an Iterative Source Method (ISM). The method is to provide an initial distribution for the source term $W$ of the fast flux equation becoming the equation system decoupled. Applying the Laplace Transform (LT) in the first equation of (1) we have the fast-flux equation transformed below:

$$
s \tilde{\phi}(x, s)-\phi_{1}(x, 0)=\left[D_{1} \frac{d^{2}}{d x^{2}} v_{1}-v_{1} \Sigma_{r 1}+(1-\beta) v_{1} \Sigma_{f 1} v_{1}\right] \tilde{\phi}(x, s)+\hat{W}(x, s)
$$

The constants $\kappa_{1}$ and $\kappa_{2}$ are obtained by replacing the boundary conditions given in (2).

$$
\tilde{\phi}_{1}(x, s)=\kappa_{1} e^{\sqrt{\gamma_{1}(s)} x}+\kappa_{2} e^{-\sqrt{\gamma_{1}(s)} x}+F_{1} x^{3}+G_{1} x^{2}+H_{1} x+I_{1}
$$

where $\gamma_{1}(s)$ is given by:

$$
\gamma_{1}(s)=\left[\frac{1}{D_{1} v_{1}} s+\frac{\Sigma_{r 1}}{D_{1}}-\frac{(1-\beta) v_{1} \Sigma_{f 1}}{v_{1}}\right]
$$

Therefore, we have $\kappa_{1}$ and $\kappa_{1}$ equal to:

$$
\kappa_{1}=\left[\frac{F_{1} e^{-\sqrt{\gamma_{1}(s)} L}-I_{1} L^{3}-H_{1} L^{2}-G_{1} L-F_{1}}{e^{\sqrt{\gamma_{1}(s)} L}-e^{-\sqrt{\gamma_{1}(s)} L}}\right]
$$


and

$$
\kappa_{2}=\left[-\kappa_{1}-F_{1}\right]
$$

Substituting the constants in the fast flux equation has the transformed equation written as:

$$
\begin{aligned}
& \tilde{\phi}_{1}(x, s)=\left[\frac{F_{1} e^{-\sqrt{\gamma_{1}(s)} L}-I_{1} L^{3}-H_{1} L^{2}-G_{1} L-F_{1}}{e^{\sqrt{\gamma_{1}(s)} L}-e^{-\sqrt{\gamma(s)_{1}} L}}\right] e^{\sqrt{\gamma_{1}(s)} x} \\
& +\left[-\left(\frac{F_{1} e^{-\sqrt{\gamma_{1}(s)} L}-I_{1} L^{3}-H_{1} L^{2}-G_{1} L-F_{1}}{e^{\sqrt{\gamma_{1}(s)} L}-e^{-\sqrt{\gamma_{1}(s)} L}}\right)-F_{1}\right] e^{-\sqrt{\gamma_{1}(s)} x}+F_{1} x^{3}+G_{1} x^{2}+H_{1} x+I_{1}
\end{aligned}
$$

To solve (8) a numerical inversion has been used through the Stehfest algorithm (HASSANZA$\mathrm{DEH}, 2007)$. After the numerical inversion, a polynomial interpolation of the form:

$$
p_{j}(x, t)=y_{1}+y_{2} x+y_{3} x^{2}+y_{4} t+y_{5} x t+y_{6} x^{2} t
$$

where $y_{1}, y_{2}, y_{3}, y_{4}, y_{5}, y_{6}$ are the coefficients of the interpolated polynomial.

Substituting $\phi_{1}(x, t)$ and applying the Laplace Transform technique in the second equation of (1) we can solve the thermal flux equation given by

$$
\frac{d^{2}}{d x^{2}} \tilde{\phi}_{2}(x, s)-\tilde{\phi}_{2}(x, s)\left[\frac{s}{D_{2} v_{2}}+\frac{\Sigma_{r 2}}{D_{2}}\right]+\frac{\phi_{2}(x, 0)}{D_{2} v_{2}}+\frac{\Sigma_{s 12} \tilde{\phi}_{1}}{D_{2}}=0 .
$$

Therefore, $\tilde{\phi}_{2}(x, s)$ has solution given as follows:

$$
\tilde{\phi}_{2}(x, s)=\kappa_{3} e^{\sqrt{\gamma_{1}(s)} x}+\kappa_{4} e^{-\sqrt{\gamma_{1}(s)} x}+F_{2} x^{3}+G_{2} x^{2}+H_{2} x+I_{2},
$$

where $\gamma_{2}(s)$ is given by:

$$
\gamma_{2}(s)=\left[\frac{1}{D_{2} v_{2}} s+\frac{\Sigma_{a 2}}{D_{2}}\right] .
$$

The constants $\kappa_{3}$ and $\kappa_{4}$ are determined analogously to the constants $\kappa_{1}$ and $\kappa_{2}$. Thus, the transformed thermal flux can be written as: 


$$
\begin{aligned}
& \tilde{\phi}_{2}(x, s)=\left[\frac{F_{2} e^{-\sqrt{\gamma_{2}(s)} L}-I_{2} L^{3}-H_{2} L^{2}-G_{2} L-F_{21}}{e^{\sqrt{\gamma_{2}(s)} L}-e^{-\gamma_{2}(s) L}}\right] e^{\sqrt{\gamma_{2}(s)} x} \\
& +\left[-\left(\frac{F_{2} e^{-\sqrt{\gamma_{2}(s)} L}-I_{2} L^{3}-H_{2} L^{2}-G_{2} L-F_{2}}{e^{\sqrt{\gamma_{2}(s)} L}}-e^{-\sqrt{\gamma_{2}(s)} L}\right)-F_{2}\right] e^{-\sqrt{\gamma_{2}(s)} x}+F_{2} x^{3}+G_{2} x^{2}+H_{2} x+I_{2}
\end{aligned}
$$

By applying the Laplace Numerical Inverse Transform in (13) and interpolating in a polynomial with the same format of (9) we can solve the equations of the concentrations given by (1). Replacing $\phi_{1}(x, t)$ and $\phi_{2}(x, t)$ into the equations of concentration and applying the Laplace Transform technique we have:

$$
s \hat{C}_{i}(x, s)+\lambda_{i} \hat{C}_{i}(x, s)=C_{i}(x, 0)+\left[\beta_{i} v_{1} \Sigma_{f 1}\right] \tilde{\phi}_{2}(x, s)+\left[\beta_{i} v_{2} \Sigma_{f 2}\right] \tilde{\phi}_{2}(x, s),
$$

Where $C_{i}(x, 0)$ are obtained as follows:

$$
C_{i}(x, 0)=\frac{\beta_{i}\left[v_{1} \Sigma_{f 1} \phi_{1}(x, 0)+v_{2} \Sigma_{f 2} \phi_{2}(x, 0)\right]}{\lambda_{i}},
$$

where $i=1: 6$.

Similarly, the methodology used for the fast and thermal fluxes can be solved (14) by applying the Numerical Inverse Laplace Transform and interpolating the solutions of the concentrations in the same polynomial form given by (9).

Then the source term $\mathrm{W}$ of the fast flux equation is updated (1) with the fluxes $\phi_{1}^{[k]}, \phi_{2}^{[k]}, C_{1}^{[k]}, C_{2}^{[k]}, C_{3}^{[k]}, C_{4}^{[k]}, C_{5}^{[k]}$ and $C_{6}^{[k]}$ being $k$ the index representing the iteration number.

Therefore, we can solve the system of equations of (1) through an iterative process described as follows:

1. Estimate of $\phi_{1}^{[k]}, \phi_{2}^{[k]}, C_{1}^{[k]}, C_{2}^{[k]}, C_{3}^{[k]}, C_{4}^{[k]}, C_{5}^{[k]}$ and $C_{6}^{[k]}$;

2. The fast-flux equation is solved;

a. Apply to LT in the fast flux equation;

b. Apply to Numerical Inverse Laplace Transform;

c. The flux is interpolate.

3. The thermal flux equation is solved; 
a. Replaces $\phi_{1}(x, t)$ Interpolated in the thermal flux equation;

b. Apply to LT in the thermal flux equation;

c. Apply to Numerical Inverse Laplace Transform;

d. The flux is interpolate.

4. Solve the equations of the concentrations;

a. Replaces $\phi_{1}(x, t)$ e $\phi_{2}(x, t)$ Interpolated in the concentration equations;

b. Apply to LT in the equations of concentrations;

c. Apply to Numerical Inverse Laplace Transform;

d. The concentrations are interpolate.

5. Stop criterion test $\frac{\left|\phi_{j}^{[k+1]}-\phi_{j}^{[k]}\right|}{\phi_{j}^{[k+1]}}<\varepsilon$;

6. If the stop criterion is satisfied the process is terminated otherwise the index $\mathrm{k}$ is updated is returned to item 1 .

\section{RESULTS AND DISCUSSION}

To validate the methodology proposed in this work we were used an algorithm implemented in the Scilab platform applied to a problem with two groups of energy and six groups of delayed neutron precursors with domain $0 \leq x \leq 160 \mathrm{~cm}, k_{\text {eff }}=0,8520306528$ and nuclear parameters given by the tables 1 and 2.

Table 1: Nuclear Parameters.

\begin{tabular}{ccc}
\hline Parameters & Group 1 & Group 1 \\
\hline$D[\mathrm{~cm}]$ & 1,0 & 0,5 \\
$v[\mathrm{~cm} / \mathrm{s}]$ & $1,0 \times 10^{-7}$ & $3,0 \times 10^{5}$ \\
$\Sigma_{a}\left[\mathrm{~cm}^{-1}\right]$ & 0,02 & 0,08 \\
$v \Sigma_{f}\left[\mathrm{~cm}^{-1}\right]$ & 0,005 & 0,099 \\
$\Sigma_{1 \rightarrow 2}\left[\mathrm{~cm}^{-1}\right]$ & 0,01 & 0 \\
\hline
\end{tabular}


Table 2: Parameters related to delayed neutrons.

\begin{tabular}{ccc}
\hline$i$ & $\beta_{i}$ & $\lambda_{1}\left[\mathrm{~s}^{-1}\right]$ \\
\hline 1 & 0,00025 & 0,0124 \\
2 & 0,00164 & 0,0305 \\
3 & 0,00147 & 0,1110 \\
4 & 0,00296 & 0,3010 \\
5 & 0,00086 & 1,1400 \\
\hline
\end{tabular}

As the initial condition the solution was used for the steady-state diffusion equation solved by (ZANETTE, 2017). The Tables 3 and 4 show the numerical convergence of the fast and thermal flows in $t=1 \mathrm{~s}$ and $80 \mathrm{~cm}$ with the decrease of $\varepsilon$, respectively. In the Table 3 to 4 equal to $10^{-5}$ we have a 6-digit concordance comparing with $10^{-8}$, already for $10^{-7}$ has an 8-digit agreement, which indicates that as $\varepsilon$ tends to zero the solution tends to stabilize. It can be observed in Table 4 .

Table 3: Numerical stability of the fast neutron flux.

\begin{tabular}{cc}
\hline$\varepsilon$ & $\phi_{1}\left[\mathrm{~cm}^{-2} \mathrm{~s}^{-1}\right]$ \\
\hline $10^{-3}$ & 0,000905058 \\
$10^{-5}$ & 0,000889528 \\
$10^{-6}$ & 0,000888144 \\
$10^{-7}$ & 0,000888022 \\
$10^{-8}$ & 0,000888010 \\
\hline
\end{tabular}


Table 4: Numerical stability of the thermal neutron flux.

\begin{tabular}{cc}
\hline$\varepsilon$ & $\phi_{2}\left[\mathrm{~cm}^{-2} \mathrm{~s}^{-1}\right]$ \\
\hline $10^{-3}$ & 0,000111869 \\
$10^{-5}$ & 0,000109938 \\
$10^{-6}$ & 0,000109766 \\
$10^{-7}$ & 0,000109751 \\
$10^{-8}$ & 0,000109749 \\
\hline
\end{tabular}

In the Table 5 we have the values of the fast and thermal fluxes for various times. As the stopping criterion of the algorithm was used $\varepsilon=10^{-8}$ for both the fast flux and the thermal flux. For this case, the maximum allowed error was $10^{-8}$ in $x=80 \mathrm{~cm}$.

Table 5: Parameters related to delayed neutrons.

\begin{tabular}{ccc}
\hline time $(\mathrm{s})$ & $\phi_{1}\left[\mathrm{~cm}^{-2} \mathrm{~s}^{-1}\right]$ & $\phi_{2}\left[\mathrm{~cm}^{-2} \mathrm{~s}^{-1}\right]$ \\
\hline 1 & 0,000879889 & 0,000108745 \\
2 & 0,000848293 & 0,000104841 \\
3 & 0,000816697 & 0,000100937 \\
4 & 0,000785101 & 0,000097032 \\
5 & 0,000753505 & 0,000093128 \\
6 & 0,000721908 & 0,000089223 \\
7 & 0,000690313 & 0,000085319 \\
8 & 0,000658716 & 0,000081414 \\
9 & 0,000627120 & 0,000077510 \\
10 & 0,000595524 & 0,000073606 \\
\hline
\end{tabular}

In the Figures 1 and 2 we have the graph of the fast and thermal flux respectively as a function of the spatial variable. 
Figure 1: Fast Neutron Flux

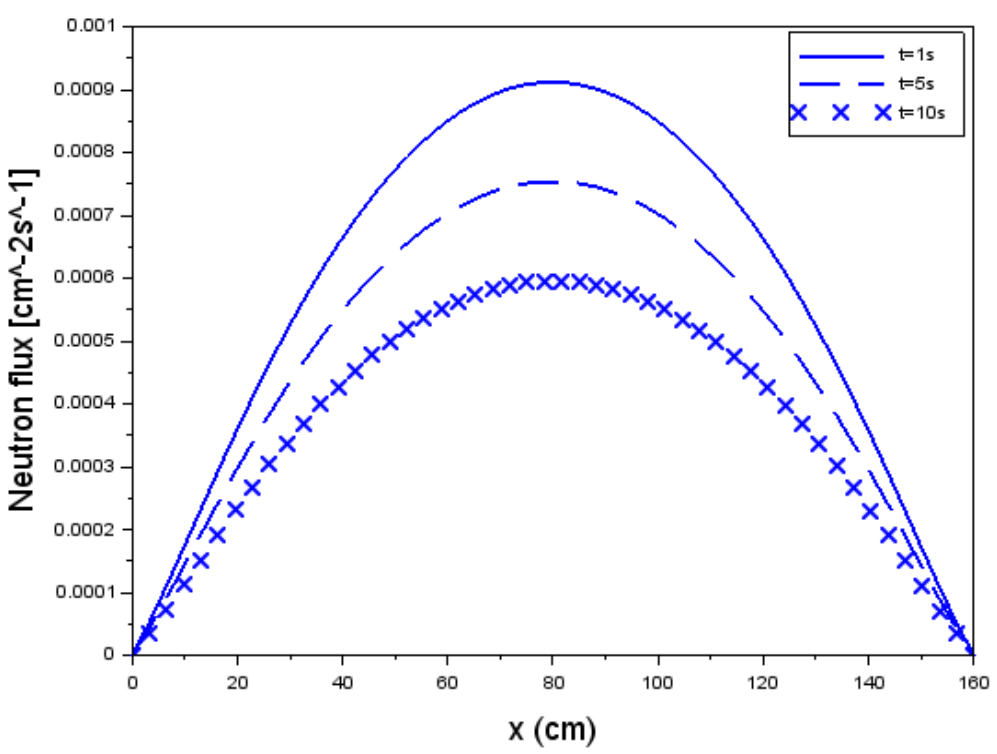

Figure 2: Thermal Neutron Flux

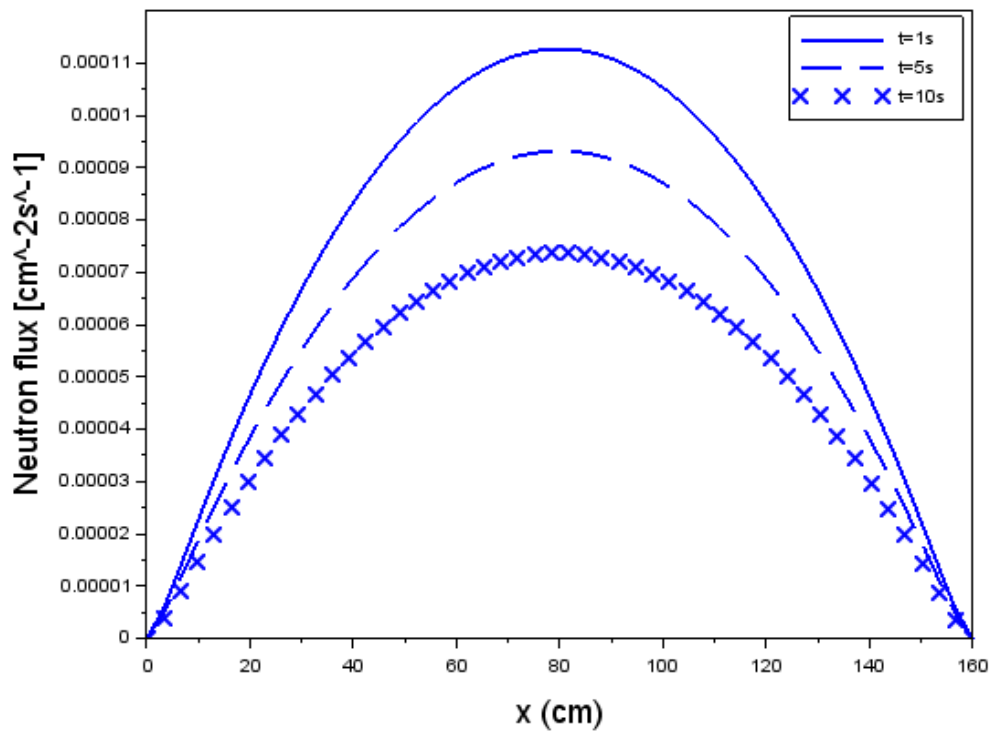




\section{CONCLUSION}

Analyzing the previous results, it can be observed that the methodology proposed in this article is promising and important in the solution of neutron space kinetics problems. Through the source iterative method, the system equations become decoupled which allows them were resolved separately becoming the solution of the problem simpler. Another important characteristic with respect to the methodology proposed is the achievement of a semi-analytic solution at each iteration. As future perspectives for the work, it is intended to apply the same methodology to the multi-region problem and to analyze the convergence of the method.

\section{ACKNOWLEDGMENT}

We would like to thank CAPES - Coordination of Superior Level Staff Improvement for supported this work.

\section{REFERENCES}

ADAMS, M.; LARSEN, E. Fast iterative methods for discrete ordinates particle transport calculations. Progress in Nuclear Energy, v. 40, p. 3-159, 2002.

CEOLIN, C.; et al. On the analytical Solution of the multi group neutron diffusion kinetic equation in a multilayered slab. International Nuclear Atlantic Conference, 2011, Belo Horizonte, MG, Brazil, October 24 to October 28 (2011).

CORNO, S. et al. Analytical approach to the neutron kinetics of the non-homogeneous reactor. Progress in Nuclear Energy, v. 50, p. 847-865, 2008.

DANIELLA, M. Métodos de Aceleração para a Solução da Equação de Transporte. 2017. Tese de Doutorado - PPGMAP/UFRGS, Porto Alegre/RS. 
HASSANZADEH, H.; POOLDADI-DARVISH, M. Comparison of different numerical Laplace inversion methods for engineering applications. Applied mathematics and computation, v. 189, p. 1966-1981, 2007.

NAHLA, A.; et al. Numerical Techniques for the Neutron Diffusion Equations in the Nuclear Reactors. Adv. Studies Theor. Physics, v. 6, p.649-664, 2012.

WILLERT, J.; TAITANO, T; KNOLL, D. Leveraging Anderson acceleration for improved convergence of iterative solutions to transport systems. Journal of Computational Physics, v. 273, p. 122132, 2014.

ZANETTE, R. Solução da Equação de Difusão de Nêutrons Multigrupo Multirregião Estacionária em Geometria Cartesiana pelo Método da Potência via Fronteiras Fictícias. 2017. Dissertação de Mestrado - PPGMAT/UFPEL, Pelotas/RS. 